AIAA 2001-2641

\title{
A VORTEX PARTICLE METHOD FOR COMPRESSIBLE FLOWS
}

\author{
Jeff Eldredge* Tim Colonius Anthony Leonard \\ Division of Engineering and Applied Science \\ California Institute of Technology \\ Pasadena, CA 91125
}

\begin{abstract}
A vortex particle method for the simulation of twodimensional compressible flows is developed. The computational elements are Lagrangian particles that carry vorticity, dilatation, enthalpy, entropy and density. The velocity field is decomposed into irrotational and solenoidal parts, which allows its calculation in terms of the particles' vorticity and dilatation. The particle coverage is truncated and incident acoustic waves are absorbed using a suitable boundary treatment. A Kirchhoff surface formulation is developed for computing the far-field sound. The method is applied to a co-rotating vortex pair and the results are discussed.
\end{abstract}

\section{INTRODUCTION}

Vortex methods have matured into useful tools for the high-fidelity simulation of incompressible flows. ${ }^{1,10}$ Particles carry vorticity and convect with the local flow velocity. The goal of the present work is to adapt vortex methods for use in compressible flows, particularly those in which sound is generated.

A computational method whose basic elements are vorticity-laden particles is a natural tool with which to simulate flows that generate sound. It has been known that vorticity is intrinsic to the production of sound since the work of Powell. ${ }^{17}$ Several researchers $^{8,13,17}$ have identified $\nabla \cdot(\mathbf{u} \times \omega)$ as source term for a wave equation, and Möhring ${ }^{14}$ has developed an integral expression where the acoustic field is linear in the vorticity in certain cases.

The details of the method, which we refer to as the dilating vortex particle method (DVPM), will be discussed in Section 2. This discussion will include the calculation of the velocity field and the solution of the compressible equations of motion using Lagrangian particles. Also, the issue of simulating a flow in an infinite domain with a finite region of particle coverage is dealt with, as are the topics of fast

\footnotetext{
*email: eldredge@caltech.edu

Copyright (c) 2001 by the authors. Published by the American Institute of Aeronautics and Astronautics, Inc. with permission.
}

summation of the velocity field and redistribution of distorted particle configurations. The computation of the acoustic far-field using a Kirchhoff surface and the equation of Möhring is addressed in Section 3. The method is applied to a model problem in Section 4 and the results are discussed.

\section{Details}

\subsection{Velocity decomposition}

We decompose the velocity field into irrotational and solenoidal components, $\mathbf{u}=\mathbf{u}_{s}+\mathbf{u}_{i r}$, each derived from a potential: $\mathbf{u}_{s} \equiv \nabla \times \mathbf{A}$ and $\mathbf{u}_{i r} \equiv \nabla \varphi$. If $\mathbf{A}$ is chosen to be solenoidal, then the curl and the divergence of the velocity leads to, respectively,

$$
\begin{aligned}
& \nabla^{2} \mathbf{A}=-\nabla \times \mathbf{u} \equiv-\omega, \quad \text { and } \\
& \nabla^{2} \varphi=\nabla \cdot \mathbf{u} \equiv \theta
\end{aligned}
$$

When equations (1) are inverted using the Green's function for the negative Laplacian, $G$, the results are

$$
\begin{aligned}
& \mathbf{A}=G \star \omega, \quad \text { and } \\
& \varphi=-G \star \theta,
\end{aligned}
$$

where $\star$ denotes convolution and an arbitrary harmonic component has been ignored. The resulting velocity field is

$$
\mathbf{u}=(\mathbf{K} \times) \star \omega-\mathbf{K} \star \theta
$$

where $\mathbf{K} \equiv \nabla G$. The first term is the Biot-Savart integral, and the second is its dilatational counterpart.

As in the incompressible vortex particle method, the vorticity field is approximated by a set of regularized particles, or "blobs", of vorticity. In the present method these blobs will also carry dilatation to approximate this field. The particle representations of the two fields are

$$
\begin{aligned}
\widetilde{\omega}(\mathbf{x}, t) & =\sum_{p} \Gamma_{p}(t) \zeta_{\varepsilon}\left(\mathbf{x}-\mathbf{x}_{p}(t)\right), \quad \text { and } \\
\widetilde{\theta}(\mathbf{x}, t) & =\sum_{p} Q_{p}(t) \zeta_{\varepsilon}\left(\mathbf{x}-\mathbf{x}_{p}(t)\right),
\end{aligned}
$$


where $\Gamma_{p}=V_{p} \omega\left(\mathbf{x}_{p}\right)$ and $Q_{p}=V_{p} \theta\left(\mathbf{x}_{p}\right)$. The function $\zeta_{\varepsilon}$ is the blob function scaled by $\varepsilon$, the radius of the blob- $\zeta_{\varepsilon}(\mathbf{x})=\zeta(\mathbf{x} / \varepsilon) / \varepsilon^{d}$, where $d$ is the physical dimension. The positions and volumes of the particles are, respectively, $\left\{\mathbf{x}_{p}(t)\right\}$ and $\left\{V_{p}(t)\right\}$. The particles will translate according to the local fluid velocity,

$$
\frac{d \mathbf{x}_{p}}{d t}=\mathbf{u}\left(\mathbf{x}_{p}\right)
$$

and their volume will change according to the local fluid dilatation,

$$
\frac{d V_{p}}{d t}=Q_{p}
$$

The approximations (4) and (5) are introduced to the velocity expression and serve to desingularize the velocity kernels. Thus, the ODE that governs the particle positions is

$$
\begin{aligned}
\frac{d \mathbf{x}_{p}}{d t}= & \sum_{q} \mathbf{K}_{\varepsilon}\left(\mathbf{x}_{p}-\mathbf{x}_{q}\right) \times \Gamma_{q}(t) \\
& -\sum_{q} Q_{q}(t) \mathbf{K}_{\varepsilon}\left(\mathbf{x}_{p}-\mathbf{x}_{q}\right),
\end{aligned}
$$

where $\mathbf{K}_{\varepsilon}=\mathbf{K} \star \zeta_{\varepsilon}$ is the smoothed velocity kernel. From hereon the flow will be assumed twodimensional, so the vorticity has only a single component. The Green's function is $G=-\frac{1}{2 \pi} \log |\mathbf{x}|$ and the velocity kernel is

$$
\mathbf{K}(\mathbf{x})=-\frac{\mathbf{x}}{2 \pi|\mathbf{x}|^{2}} .
$$

Accounting for the radial symmetry of the blob function, we obtain

$$
\mathbf{K}_{\varepsilon}(\mathbf{x})=-\frac{\mathbf{x}}{2 \pi|\mathbf{x}|^{2}} q(|\mathbf{x}| / \varepsilon)
$$

where $q(r) \equiv 2 \pi \int_{0}^{r} \tau \zeta(\tau) d \tau$. For convergence of this method, ${ }^{7}$ it is necessary that the particles overlap each other (i.e. the ratio of blob radius to interparticle spacing, $\kappa=\varepsilon / \Delta x$, is greater than unity). Part of the error in (8) is related to the order of accuracy of the blob function. In the present method we use an 8th-order-accurate function (see Section 4).

\subsection{Governing equations}

The vorticity and dilatation strengths of the particles evolve according to the compressible equations of motion. To ensure some compactness of their form, the equations are expressed in terms of the specific enthalpy $(h)$, specific entropy $(s)$ and density $(\rho)$ as follows:

$$
\begin{aligned}
\frac{D \omega}{D t}= & -\omega \theta+\nabla \times(h \nabla s) \cdot \hat{\mathbf{e}}_{3} \\
& +\frac{1}{\operatorname{Re}} \nabla \times\left[\frac{1}{\rho}\left(\frac{4}{3} \nabla \theta-\nabla \times \omega\right)\right] \cdot \hat{\mathbf{e}}_{3}(11) \\
\frac{D \theta}{D t}= & -\nabla^{2} h-\nabla \mathbf{u}:(\nabla \mathbf{u})^{T}+\nabla \cdot(h \nabla s) \\
& +\frac{1}{\operatorname{Re}} \nabla \cdot\left[\frac{1}{\rho}\left(\frac{4}{3} \nabla \theta-\nabla \times \omega\right)\right], \\
\frac{D s}{D t}= & \frac{1}{\operatorname{Re}} \frac{\Phi}{\rho h}+\frac{1}{\operatorname{RePr}} \frac{1}{\rho h} \nabla^{2} h, \\
\frac{D \rho}{D t}= & -\rho \theta .
\end{aligned}
$$

where $\Phi$ is the viscous dissipation, expressed as

$$
\Phi=\omega^{2}+2 \nabla \mathbf{u}:(\nabla \mathbf{u})^{T}-\frac{2}{3} \theta^{2} .
$$

An acoustic scaling has been used to nondimensionalize the variables in terms of the ambient speed of sound, $a_{\infty}$, and density, $\rho_{\infty}$; the dynamic viscosity, $\mu$; the thermal conductivity, $k$; the specific heat at constant pressure, $c_{p}$; and a characteristic length scale, $L$. The fluid properties have been assumed constant and uniform, and the medium to behave as a calorically perfect gas. The Reynolds number is $\operatorname{Re}=\rho_{\infty} a_{\infty} L / \mu$ and the Prandtl number is $\operatorname{Pr}=\mu c_{p} / k$.

An equation of state can be used to relate the enthalpy to the density and entropy:

$$
h=\frac{\rho^{\gamma-1}}{\gamma-1} e^{\gamma s}
$$

where $\gamma$ is the ratio of specific heats, taken to be 1.4. Because the equations involve the enthalpy, entropy and density, the particles will also carry these quantities, and their fields will be composed as such:

$$
\tilde{f}(\mathbf{x}, t)=\sum_{p} V_{p} f\left(\mathbf{x}_{p}\right) \zeta_{\varepsilon}\left(\mathbf{x}-\mathbf{x}_{p}(t)\right)
$$

where $f$ is either $h, s$ or $\rho$.

The spatial derivatives in equations (11)-(15) are discretized using Particle Strength Exchange, or PSE, a technique first developed by Degond and Mas-Gallic ${ }^{2}$ for approximating the Laplacian in the advection-diffusion equation. The scheme can be extended to general differential operators, as shown by Eldredge et al. ${ }^{3}$ The essence of the method is the approximation of the derivative $D^{\beta}$ (where $\beta$ is a 
multi-index denoting the degree of derivative) by an integral operator,

$$
L^{\varepsilon} f(\mathbf{x})=\frac{1}{\varepsilon^{|\beta|}} \int_{\mathbb{R}^{2}}(f(\mathbf{y}) \mp f(\mathbf{x})) \eta_{\varepsilon}^{\beta}(\mathbf{x}-\mathbf{y}) d \mathbf{y},
$$

whose kernel, $\eta$, is tailored to $\beta$ with some order of accuracy. The choice of sign in (18) is determined by whether the derivative is odd or even, for reasons to be discussed shortly. The integral is discretized via a quadrature over the particles,

$$
D^{\beta} f\left(\mathbf{x}_{p}\right) \approx \frac{1}{\varepsilon^{|\beta|}} \sum_{q} V_{q}\left(f_{q} \mp f_{p}\right) \eta_{\varepsilon}^{\beta}\left(\mathbf{x}_{p}-\mathbf{x}_{q}\right),
$$

where $f_{p}=f\left(\mathbf{x}_{p}\right)$. If $|\beta| \equiv \beta_{1}+\beta_{2}$ is even, then the negative sign is chosen, and if $|\beta|$ is odd, then the positive sign is chosen. Allowing for this choice ensures the conservation of important global quantities when PSE is applied in certain equations. For instance, if (19) is used to approximate the Laplacian in the convection-diffusion equation in an unbounded domain, then the discrete volume integral, $\sum V_{p} f_{p}$, will remain constant in time.

For spatial derivatives in the velocity, the gradient operator is applied directly to the velocity kernels on the right-hand side of (8):

$$
\begin{aligned}
(\nabla \mathbf{u})_{p}= & \sum_{q} \Gamma_{q}(t) \nabla \mathbf{K}_{\varepsilon}\left(\mathbf{x}_{p}-\mathbf{x}_{q}\right) \times \hat{\mathbf{e}}_{3} \\
& -\sum_{q} Q_{q}(t) \nabla \mathbf{K}_{\varepsilon}\left(\mathbf{x}_{p}-\mathbf{x}_{q}\right) .
\end{aligned}
$$

The new kernel, $\mathbf{R}_{\varepsilon}=\nabla \mathbf{K}_{\varepsilon}$, can be written in terms of $\zeta$ and $q$ as

$$
\begin{aligned}
R_{\varepsilon}^{i j}(\mathbf{x})= & \left(-\zeta_{\varepsilon}(\mathbf{x})+\frac{q(|\mathbf{x}| / \varepsilon)}{\pi|\mathbf{x}|^{2}}\right) \frac{x_{i} x_{j}}{|\mathbf{x}|^{2}} \\
& -\frac{q(|\mathbf{x}| / \varepsilon)}{2 \pi|\mathbf{x}|^{2}} \delta_{i j} .
\end{aligned}
$$

Note that $\lim _{r->0} R_{\varepsilon}^{i j}(r)=\frac{1}{2} \zeta_{\varepsilon}(0) \delta_{i j}$.

Material derivatives in the continuous equations are replaced by ordinary time derivatives of the particle strengths. The particle forms of equations (11) and (12) are reformulated in terms of the integral values of vorticity and dilatation: the particle circulation, $\Gamma_{p}$, and source strength, $Q_{p}$. This approach, when combined with (7), eliminates the $\theta$ term from equation (11) and an analogous term embedded in the double contraction term in equation (12). Equations (7), (8) and the particle form of equation (13) are also solved. Given the initial density of a particle, the density at subsequent times is calculated through $\rho_{p}(t)=\rho_{p}(0) V_{p}(0) / V_{p}(t)$. This treatment explicitly ensures that the method conserves mass. The enthalpy of a particle is computed using the particle form of (16). The particle evolution equations may be solved simultaneously using a standard time integration scheme, such as the fourth-order RungeKutta method.

Also needed are initial conditions for the primary variables. Often it may be sufficient to set the initial dilatation and entropy to zero (unless the circumstances of a particular problem require otherwise), and then compute the initial enthalpy from the initial vorticity (or velocity, rather) through a solution of the following Poisson problem:

$$
\nabla^{2} h=-\nabla \mathbf{u}_{s}:\left(\nabla \mathbf{u}_{s}\right)^{T}
$$

This effectively reduces the magnitude of the transient that results from not specifying these initial conditions in an exactly consistent manner. With the initial enthalpy and entropy of a particle specified, the initial particle density can then be computed through (16):

$$
\rho_{p}(0)=\left[(\gamma-1) h_{p}(0)\right]^{1 /(\gamma-1)} \exp \left(-\frac{\gamma}{\gamma-1} s_{p}(0)\right) .
$$

The particles are initially located on a uniform Cartesian grid, with $V_{p}(0)=\Delta x^{2}$, where $\Delta x$ is the particle spacing.

\subsection{Spatial compactness}

Compressible flows inherently contain radiated components. If this radiation is to be accomodated in a numerical method by the exchange of strengths described above, then some means must be provided that allows the outer particles to exchange strength with an infinite region exterior to the particles. Furthermore, the integral for computing the irrotational component of the velocity (the second term of equation (3)) must be addressed, because its integrand does not confine itself to the partical coverage.

We note that in general it may be possible to retain some measure of the spatial compactness associated with incompressible vortex methods in the extension to compressible flow. Instead, we propose here a simpler, but likely more expensive approach, wherein particles are retained to a somewhat larger distance into the acoustic field and a non-reflecting boundary condition is enforced to absorb incident acoustic waves. Further refinements of the domain truncation will be proposed in future work. 
The Engquist-Majda ${ }^{5}$ hierarchy of conditions are used, of which the first member is

$$
\left(\frac{\partial f}{\partial t}+\frac{\partial f}{\partial r}+\frac{f}{2 r}\right)_{r=R}=0,
$$

where $R$ is the radius of curvature of the boundary and $f$ is any quantity governed by the linear wave equation. A means must be developed for enforcing the condition. We create a new class of boundary particles, which lie on the periphery of the coverage in a "boundary zone", that has a different set of properties from the class of interior particles. The new particles translate as the others do (albeit with a very small motion), but their enthalpy, $h_{p}$, is governed by

$$
\frac{d h_{p}}{d t}=-\left(\frac{\partial h}{\partial r}\right)_{p}-\frac{\left(h_{p}-h_{\infty}\right)}{2 R_{p}}
$$

instead of (the particle form of) equation (16), where $R_{p}$ is the radial distance of the particle from the origin. Their dilatation, vorticity (which is zero by assumption) and entropy are held constant. The boundary zone has a depth that depends on the blob radius of the particles; typically only a few particles' depth is required.

The spatial derivative in (24) is approximated using PSE. However, the technique of approximating the derivative by a full-space integral is inaccurate. A kernel centered at a point near the boundary expects to have information available from an approximately circular region surrounding the point. If any portion of this region fails to intersect with the computational domain, then the subsequent quadrature of the integral will be poor. In these cases an integral over the "inner half-space" is used in lieu of the full-space version, ${ }^{3}$ and its quadrature will only involve particles located in a half-disc corresponding to the intersection of the kernel support with the half-space. The error from such a treatment can be shown to be dominated by the incomplete annihilation of a cylindrical wave by equation (23); for a general wave the leading-order error is $O\left(R^{-5 / 2}\right)$.

For the dilatation term in equation (3), the integration is simply truncated at the boundary of particle coverage. For most flows of interest, the irrotational component is significantly smaller than the solenoidal, and furthermore the dilatation external to the domain is likely to contribute very little to this small irrotational part.

\subsection{Fast summation}

The velocity computation in (8) is inherently $O\left(N^{2}\right)$, which would prohibit simulations using more than a few thousand particles. Several fast summation methods have been developed for reducing this calculation to $O(N \log N)$ or $O(N)$. The Fast Multipole Method (FMM) of Greengard and Rokhlin ${ }^{6}$ treats the particles as monopoles and lumps their far-field interactions into interactions between clusters, using formulas that shift the centers of the multipole expansions. The FMM is adapted to the present method by regarding the particles as vortexsource superpositions. The reader is referred to Eldredge et al. ${ }^{4}$ for details.

\subsection{Remeshing}

Because of the nature of its topology, the flow will often contain regions of high accumulated strain where fluid elements have been stretched. Such behavior is reflected in those regions becoming sparsely populated with computational elements, thus violating the convergence requirement of vortex methods that the blobs overlap. Particles must periodically be redistributed to prevent such accumulation. Interpolation of this type has proved an essential part of $\mathrm{SPH}^{15}$ and vortex particle methods. ${ }^{1}$ Extra care must be taken when interpolating in the present method because of the sensitivity of the acoustic field to even the smallest errors. Through trial and error, we have found that interpolation can degrade the smoothness of the enthalpy and introduce an error which is subsequently amplified by PSE, overwhelming (12) unless higher-order interpolation with some degree of smoothing is used. ${ }^{4}$ Through experience we have found that a 6th-order kernel works well for interpolation:

$$
\begin{aligned}
W\left(x_{1}, x_{2}\right)= & \frac{1}{\pi}\left(\frac{15}{8}-\frac{5 x_{1}^{2}}{2}+\frac{x_{1}^{4}}{2}\right) \\
& \times\left(\frac{15}{8}-\frac{5 x_{2}^{2}}{2}+\frac{x_{2}^{4}}{2}\right) e^{-\left(x_{1}^{2}+x_{2}^{2}\right)} .
\end{aligned}
$$

The particle vorticity, dilatation, density and entropy are interpolated every one or two steps onto a new set of particles using

$$
\tilde{f}_{p}=\sum_{q} W_{\sigma}\left(\tilde{\mathbf{x}}_{p}-\mathbf{x}_{q}\right) V_{q} f_{q}
$$

where $\left\{f_{q}\right\},\left\{\mathbf{x}_{p}\right\}$ and $\left\{V_{p}\right\}$ are the old particle strengths, positions and volumes, and $\left\{\tilde{f}_{q}\right\}$ and $\left\{\tilde{\mathbf{x}}_{p}\right\}$ are the new particle strengths and positions. The kernel is scaled by the interpolation radius, $W_{\sigma}(\mathbf{x})=$ $W(\mathrm{x} / \sigma) / \sigma^{2}$, where $\sigma=1.7 \Delta x$. 


\section{The ACOUstic FiEld}

In the far-field, variables such as the pressure and enthalpy are governed by the homogeneous wave equation. The solution in this so-called "acoustic" region can be determined in one of three ways: the extension of particle coverage to this region, a Kirchhoff surface, or an acoustic analogy. The first is impractical for low Mach number flows unless particles of varying size are incorporated into the method for efficient resolution of the disparate length scales, a feature still under development. Thus, in the present work we use the latter two, and for the last we use the analogy of Möhring. ${ }^{14}$

\subsection{Kirchhoff surface}

Provided that the computational domain extends into the acoustic region, the entire far-field solution can be deduced from the limited acoustic information available from the near-field simulation through the use of a Kirchhoff surface. For a field quantity, $f$, governed by the linear, homogeneous wave equation in $\Omega_{e}$, the Kirchhoff equation expresses its solution in this domain in terms of its boundary and initial values.

In two dimensions, provided that the initial values of $f$ and its derivative are zero in the exterior domain and on its interior boundary $\partial \Omega_{e}$, the solution $f(\mathbf{x}, t)$ in $\Omega_{e}$ can be expressed as: ${ }^{4}$

$$
\begin{aligned}
f(\mathbf{x}, t)= & \frac{1}{2 \pi} \int_{\partial \Omega_{e}} \int_{0}^{t^{*}}\left[\left(\frac{\partial f}{\partial \tau}+\frac{f(\mathbf{y}, \tau)}{t-\tau+|\mathbf{x}-\mathbf{y}|}\right)\right. \\
& \left.\times\left(\hat{\mathbf{e}}_{R} \cdot \mathbf{n}_{y}\right)-\frac{\partial f}{\partial n_{y}}(\mathbf{y}, \tau)\right] \\
& \times \frac{d \tau d S(\mathbf{y})}{\sqrt{(t-\tau)^{2}-|\mathbf{x}-\mathbf{y}|^{2}}}
\end{aligned}
$$

The time integral is integrated to the retarded time, $t^{*}=t-|\mathbf{x}-\mathbf{y}|$. The vector $\hat{\mathbf{e}}_{R}$ is the unit vector from the source position, $\mathbf{y}$, in the direction of the observation point, $\mathbf{x} ; \mathbf{n}_{y}$ is the inward normal at $\mathbf{y}$ (or outward from the interior region).

The variable $f$ chosen determines how far one must go from the nonlinear source region to find the acoustic region. If the enthalpy is chosen, then the region in which this variable is entirely acoustic is apparently quite distant from the source. Outside of the vortical region, the enthalpy obeys Bernoulli's equation: $h=h_{\infty}-\frac{1}{2}|\mathbf{u}|^{2}-\frac{\partial \chi}{\partial t}$, where $\chi$ is the scalar potential consistent with the velocity induced by the vorticity. An asymptotic matching of the near- and far-field solutions reveals that, while an expansion of $\frac{\partial \chi}{\partial t}$ matches with the outer solution term by term, the $\frac{1}{2}|\mathbf{u}|^{2}$ term has no counterpart in the far-field, yet persists to large distances because it decays like $1 / r^{2}$. A more appropriate acoustic variable is the stagnation enthalpy, $B=h+\frac{1}{2}|\mathbf{u}|^{2}$. In the irrotational region, $B=B_{\infty}-\frac{\partial \chi}{\partial t}$, so it satisfies the acoustic equations just outside of the vortical region; in the far-field, $B$ and $h$ are equal.

The integrals of equation (26) are discretized in space and time. A circular Kirchhoff surface is used that surrounds the vortical region but is sufficiently removed from the edge of the computational domain to avoid corruption from the boundary treatment. The stagnation enthalpy of each particle is computed from the results of the DVPM simulation and the particle data is interpolated onto the surface control points. The time derivative is computed from backward differencing and the normal derivative from a PSE calculation.

\subsection{Möhring analogy}

For flows in which the vortical region is compact relative to the wavelength of sound, the Möhring acoustic analogy ${ }^{14}$ can be used to compute the farfield sound. It requires only the third time derivative of the second moments of vorticity to calculate the pressure at points outside of the source region. The expression for the pressure was adapted to twodimensional flow by Mitchell et al., ${ }^{12}$ to which we refer the reader for details.

\section{Results}

The method was applied to a pair of identical vortices in a compressible medium. As the vortices orbit each other they generate sound at a frequency of twice their rotation rate. The problem has been explored by several researchers (e.g. Müller and Obermeier $^{16}$ ) and recently simulated by Mitchell, Lele and Moin ${ }^{12}$ using a compact finite-difference method on a stretched grid. The vortices are initially placed at $(x, y)=(0, \pm R)$. All quantities are scaled by $R$ and the ambient speed of sound, $a_{\infty}$. For comparison with Mitchell et al., each vortex is Gaussiandistributed according to

$$
\omega=\frac{1.25 \Gamma_{0}}{\pi r_{0}^{2}} e^{-1.25 r^{2} / r_{0}^{2}}
$$

where the circulation and radius of each vortex are $\Gamma_{0}=-2 \pi(0.7)^{-1} M_{0} r_{0}$ and $r_{0}=0.15$, respectively. The circulation Reynolds number, Re $\equiv\left|\Gamma_{0}\right| / \nu$ is 7500 , the vortex Mach number, $M_{0} \equiv U_{0} / a_{\infty}$ (where $U_{0}$ is the maximum azimuthal velocity of a single Gaussian vortex) is 0.56, and the Prandtl number 

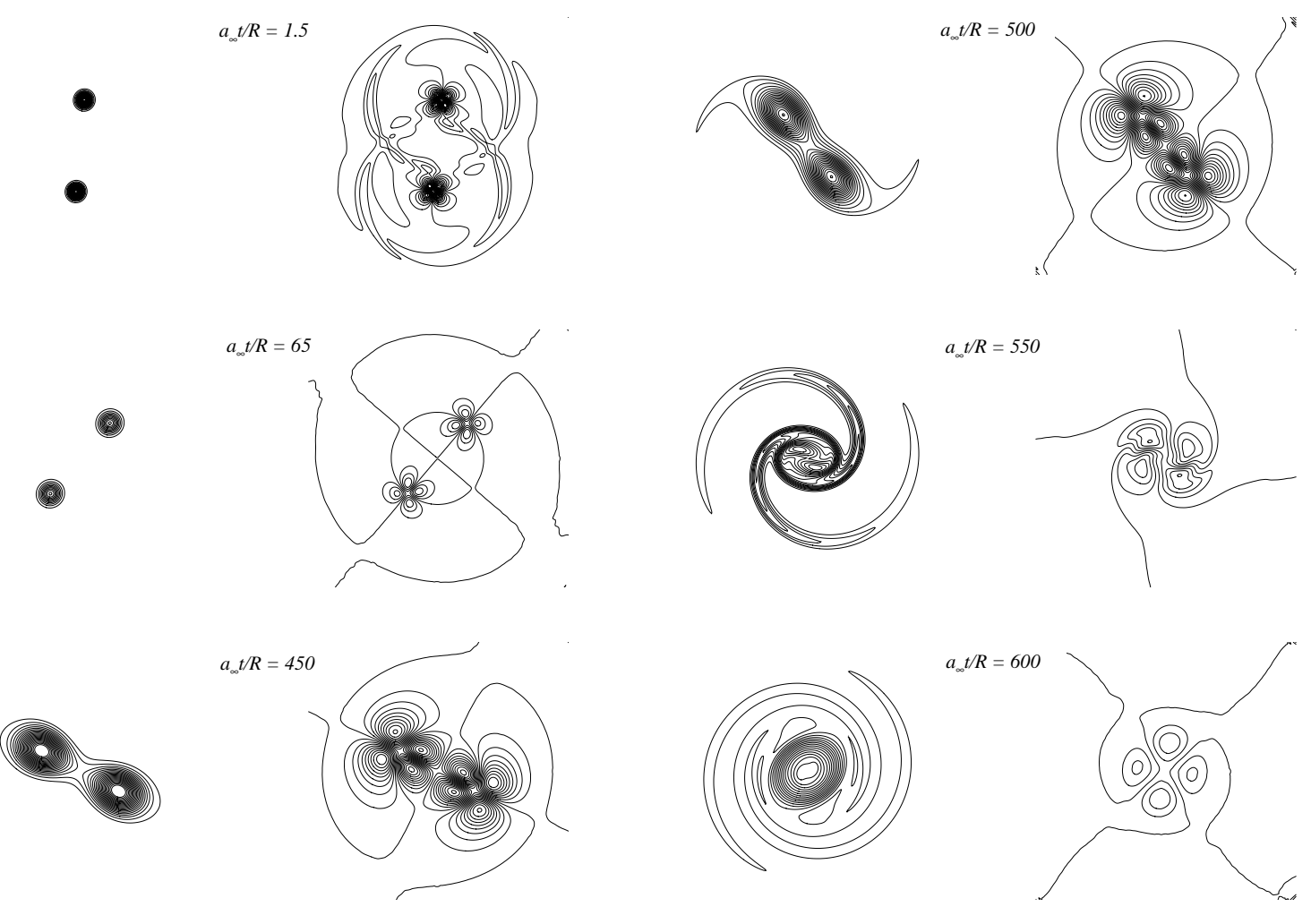

Figure 1: Vorticity (left) and dilatation (right) in co-rotating vortex problem.

is 0.7 . With these flow parameters, the initial rotation time is $\tau=105$ and the wavelength of sound is $\lambda=52.5$. Such a large separation in the acoustic and flow length scales qualifies the vortices as a compact source, which makes efficient resolution of the problem difficult. Instead of attempting to capture both the near- and far-field simultaneously, the present investigation focused on the near-field dynamics only. The method is capable of computing both, but without particles of variable size it cannot compute both regions practically.

Initially, the flow is taken as homentropic and dilatation-free. The initial enthalpy is deduced from a solution of the Poisson equation (21), which is solved with the same Green's function approach used to invert the potential equations (1). The particles are distributed on a Cartesian grid inside a circular domain of radius $R_{\Omega}$ with $N_{\text {core }}$ particles laid across the diameter of each vortex; a boundary zone with a depth of 4 particles surrounds the domain. The particles are remapped to the same Cartesian grid every $n_{r m}$ time steps. A fourth-order RungeKutta scheme is used for time advancement with a time-step size of $\Delta t=0.009$. The blob radius and particle spacing are related by $\varepsilon=\Delta x^{0.85}$. All of the kernels used in the interior are 8th order accurate, except for the one-sided boundary kernel, which is 2nd order accurate:

$$
\begin{aligned}
& \zeta(\mathbf{x})=\frac{1}{\pi}\left(4-6|\mathbf{x}|^{2}+2|\mathbf{x}|^{4}-\frac{1}{6}|\mathbf{x}|^{6}\right) e^{-|\mathbf{x}|^{2}} \\
& \eta^{l a p}(\mathbf{x})=\frac{1}{\pi}\left(40-40|\mathbf{x}|^{2}+10|\mathbf{x}|^{4}-\frac{2}{3}|\mathbf{x}|^{6}\right) e^{-|\mathbf{x}|^{2}} \\
& \eta^{(1,0)}(\mathbf{x})=-\frac{1}{\pi} x_{1}\left(20-20|\mathbf{x}|^{2}+5|\mathbf{x}|^{4}-\frac{1}{3}|\mathbf{x}|^{6}\right) e^{-|\mathbf{x}|^{2}} \\
& \eta^{L,(1,0)}(\mathbf{x})=-\frac{4}{\pi} x_{1}\left(5-2|\mathbf{x}|^{2}\right) e^{-|\mathbf{x}|^{2}}
\end{aligned}
$$

The results of the vorticity and dilatation fields from a computation with $N_{\text {core }}=13, R_{\Omega}=4$ (corresponding to about 83,500 particles), and $n_{r m}=2$ are depicted in the series of panels of Figure 1. The first row of panels shows the fields soon after the initialization. An acoustic transient is emitted from each core as the dilatation settles to the correct value; the transient is not strong and exits the domain without significant reflection. A quadrupole structure is observed in the dilatation in the next row of panels; the same structure was observed by Mitchell et al. The configurations of both fields per- 


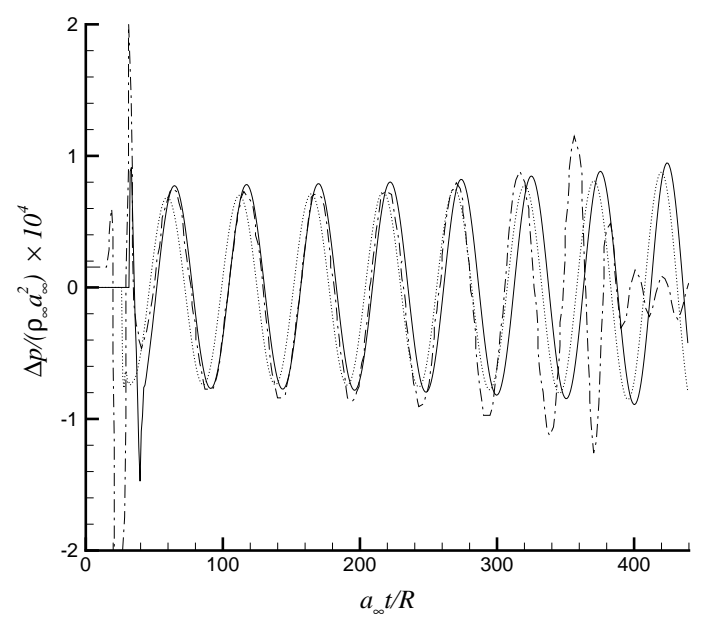

Figure 2: Pressure fluctuations observed at $(x, y)=$ $\left(0, \frac{1}{2} \lambda\right)$. Mitchell: '-.-'; DVPM and Kirchhoff surface at $r=3.5$ : '—_ ' DVPM and Möhring analogy: '......'

sist for several rotations, though both quantities are diffused by viscosity over this duration, as observed in the third set of panels (in which the contour levels have been adjusted for better resolution of the diffused magnitudes). After approximately four rotations the continual effects of viscosity and compressibility force the cores to merge, depicted in the final three rows of panels. The resulting dilatation field is a much weaker quadrupole centered at the core of the new elliptical vortex. Further computation, not shown, reveals the axisymmetrization of the core and thus the disappearance of the dilatation.

The pressure fluctuations observed at one halfwavelength from the origin (on the $y$-axis), using both the Kirchhoff surface and the Möhring formula, are depicted in Figure 2 and compared with the results of Mitchell et al. Note that because of the symmetry of the problem each rotation of the vortices corresponds to two wavelengths of sound. The magnitude and phase of the pressure agree quite well for the first two rotations. The large spike in Mitchell's data at the outset is due to the acoustic transient. Such a spike is not exhibited in the present results because of the filtering by the Möhring integral and the neglect of the initial transient period for the Kirchhoff surface. After a little more than three rotations the vortices in Mitchell's simulation merge, reflected by a small rise and then quick decay of the

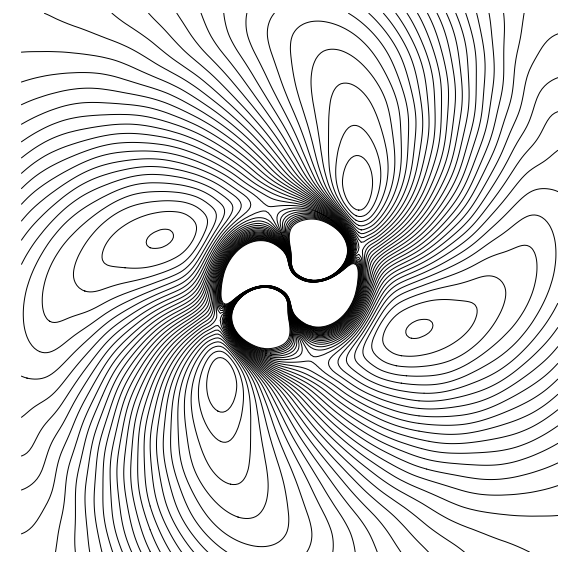

Figure 3: Dilatation field at $t=81$ for a noncompact vortex pair. The countour levels are saturated so much smaller values in the outer regions are clear.

pressure. Merger in the present simulation is delayed by an extra one-and-a-half rotations, though. As shown by Melander et al., ${ }^{11}$ incompressible vortices which are initially separated by more than the critical distance for convective merger persist in a 'metastable' state for a duration dependent upon a viscous timescale. The vortices eventually undergo convective merger, but the time at which this begins may be quite sensitive to small perturbations. Thus, it is not surprising that merger occurs later in our simulation.

The difficult task of capturing both the near-field dynamics and the far-field acoustics is alleviated when the acoustic wavelength is not so large compared to the extent of the vortical region. As a further demonstration of the capabilities of the DVPM, the same problem was simulated with larger vortex cores, $r_{0}=0.45$, which corresponds to a wavelength of $\lambda=17.5$. Larger cores permits a larger region to be covered by particles. Using the same number of particles as in the previous simulation, the computational domain was enlarged to a radius $R_{\Omega}=12$. The resulting dilatation field at $t=81$ is depicted in Figure 3, with the contour levels saturated for clarity of the acoustic field. At the time shown, the vortices are merging. It is interesting to note that Mitchell et al. did not observe merger after five full rotations, or 175 units of time, using vortices of the same size but with a much larger Reynolds number. 
The counter-clockwise tilting of the dilatation structure in the outer regions is due to the phase lag of compressibility.

\section{Conclusion}

A vortex particle method for two-dimensional compressible flow has been developed. This method is the first Lagrangian method for simulation of the full compressible Navier-Stokes equations. By using particles which are able to change volume and which carry vorticity, dilatation, enthalpy, entropy, and density, the equations of motion are satisfied. A scheme for enforcing a non-reflecting boundary condition has also been introduced and successfully implemented. The Fast Multipole Method has been adapted to compressible particles for more efficient implementation. The new vortex method has been applied to co-rotating vortices in a compressible medium, and the results agree well with those of previous work.

Because of the small relative magnitude of the acoustic field, the method requires more delicate application of techniques that have proven robust for incompressible vortex methods, for instance computation of derivatives using PSE (which must now suppress dispersion of waves) and interpolation during remeshing (which must preserve smoothness in the interpolated quantities). This subtle balance comes as no suprise, as workers in computational aeroacoustics have long been cognizant of the need for high-order methods (e.g. Lele ${ }^{9}$ ).

We believe this method shows promise, but further developments are necessary in order to solve problems of larger scale. A more "efficient" definition of the particles - for instance, a division of the particles into those which are active and those which are passive in the velocity induction - is currently being explored. Such a division would permit simulations with two different time steps when the time scales of physical phenomena in the flow are distinct. Along the same lines, an implementation of the method with variably-sized particles, which would allow more efficient resolution of flows with disparate length scales, is under development. Such an extension would make simultaneous solution of the near and acoustic fields practical. The boundary treatment proposed here is sufficient for absorbing incident acoustic waves, but does not fully exploit the decomposition of the velocity at the heart of the method. A more natural scheme is currently being developed. Finally, using existing techniques for computing vortex stretching, we believe that the method is readily extendable to three-dimensional flows.

\section{ACKNOWLEDGEMENTS}

The first author gratefully acknowledges support from a Graduate Research Fellowship from the National Science Foundation.

\section{REFERENCES}

[1] G.-H. Cottet and P. Koumoutsakos. Vortex Methods: Theory and Practice. Cambridge University Press, 2000.

[2] P. Degond and S. Mas-Gallic. The weighted particle method for convection-diffusion equations, Part 1: The case of an isotropic viscosity. Math. Comp., 53(188):485-507, 1989.

[3] J. Eldredge, T. Colonius, and A. Leonard. A general deterministic treatment of derivatives in particle methods. Submitted to SIAM J. Sci. Comput., 2001.

[4] J. Eldredge, T. Colonius, and A. Leonard. A vortex particle method for compressible flows. Submitted to J. Comput. Phys., 2001.

[5] B. Engquist and A. Majda. Absorbing boundary conditions for the numerical simulation of waves. Math. Comp., 31(139):629-651, 1977.

[6] L. Greengard and V. Rokhlin. A fast algorithm for particle simulations. J. Comput. Phys., 73(2):325-348, 1987.

[7] O. H. Hald. Convergence of vortex methods for Euler's equations, II. SIAM J. Numer. Anal., 16:726-755, 1979 .

[8] M. S. Howe. Contributions to the theory of aerodynamic sound, with application to excess jet noise and the theory of the flute. J. Fluid Mech., 71(4):625-673, 1975.

[9] S. Lele. Computational aeroacoustics: A review. AIAA Paper 97-0018, 1997.

[10] A. Leonard. Vortex methods for flow simulation. J. Comput. Phys., 37(3):289-335, 1980.

[11] M. Melander, N. Zabusky, and J. McWilliams. Symmetric vortex merger in two dimensions: causes and conditions. J. Fluid Mech., 195:303$340,1988$. 
[12] B. Mitchell, L. S.K., and M. P. Direct computation of the sound from a compressible corotating vortex pair. J. Fluid Mech., 285:181202, 1995.

[13] W. Möhring. Sound generation from convected vortices. Unpublished.

[14] W. Möhring. On vortex sound at low Mach number. J. Fluid Mech., 85:685-691, 1978.

[15] J. J. Monaghan. Particle methods for hydrodynamics. Comp. Phys. Rep., 3:71-124, 1985.

[16] E.-A. Müller and F. Obermeier. The spinning vortices as a source of sound. In Proc. Conf. on Fluid Dynamics of Rotor and Fan Supported Aircraft at Subsonic Speeds, AGARD CP-22, 1967.

[17] A. Powell. Theory of vortex sound. J. Acoust. Soc. Am., 36(1):177-195, 1964. 